\title{
Bone Healing Capacity of Demineralized Dentin Matrix Materials in a Mini-pig Cranium Defect
}

\author{
Jong-Yub Kim', Kyung-Wook Kim¹, In-Woong Um², Young-Kyun Kim³, \\ Jeong Keun Lee ${ }^{4}$
}

1. Department of Oral and Maxillofacial Surgery, College of Dentistry, Dankook University, Cheonan, Korea

2. Korea Tooth Bank, Seoul, Korea

3. Department of Oral and Maxillofacial Surgery, Section of Dentistry, Seoul National University Bundang Hospital, Seongnam, Korea

4. Department of Oral and Maxillofacial Surgery, Ajou University School of Medicine, Suwon, Korea

\begin{abstract}
- Abstract
Purpose: In this study the bone healing ability of autogenous tooth bone graft material as a substitute material was evaluated in a mini-pig cranial defect model through histologic examinations and osteonectin reverse transcription polymerase chain reaction (RT-PCR) quantitative analysis.

Materials and Methods: A defect was generated in the cranium of mini-pigs and those without a defect were used as controls. In the experimental group, teeth extracted from the mini-pig were manufactured into autogenous tooth bone graft material and grafted to the defect. The mini-pigs were sacrificed at 4,8 , and 12 weeks to histologically evaluate bone healing ability and observe the osteonectin gene expression pattern with RT-PCR.

Result: At 4 weeks, the inside of the bur hole showed fibrosis and there was no sign of bone formation in the control group. On the other hand, bone formation surrounding the tooth powder granule was observed at 4 weeks in the experimental group where the bur hole was filled with tooth powder. Osteonectin gene expression; there was nearly no osteonectin expression in the control group while active osteonectin expression was observed from 4 to 12 weeks in the experimental group.
\end{abstract}

Conclusion: We believe this material will show better results when applied in a clinical setting.

- Key words : Autogenous tooth bone, Cranium defect, Mini-pig, Reverse transcription polymerase chain reaction

- J Korean Dent Sci. 2012; 5(1) : 21 - 28

\section{Corresponding Author}

\section{Kyung-Wook Kim, DDS, PhD}

Department of Oral and Maxillofacial Surgery, College of Dentistry, Dankook University, 119 Dan-daero, Dongnamgu, Cheonan 330-714, Korea

TEL : +82-41-550-1991 3 FAX : +82-41-551-8988 E-mail : kkwoms@dku.edu

Received for publication December 8, 2011; Returned after revision May 14, 2012;

Accepted for publication May 21, 2012

(c) This is an open access article distributed under the terms of the Creative Commons Attribution Non-Commercial License (http:// creativecommons.org/licenses/by-nc/3.0) which permits unrestricted non-commercial use, distribution, and reproduction in any medium, provided the original work is properly cited. 


\section{Introduction}

Since the existence of bone morphogenetic protein (BMP) with ectopic bone formation effect and osteoinductive abilities were first reported by Urist ${ }^{1}$, and the bone formation ability of decalcified tooth dentin grafted to oral, bone, and even muscle soft tissue has been reported in line ${ }^{2)}$. Efforts to use teeth in the development of bone graft material has become active only recently with Kim et al. ${ }^{3)}$ of Korea reporting that teeth, when treated properly, can be successfully applied as graft material and Murata et al. ${ }^{4)}$ of Japan reported success in forming bone from experimentally decalcified teeth.

Considering safety and validity, autogenous bone is the ideal choice for graft material. However, extra surgery for bone harvesting and the possibility of infection and pain caused by this surgery along with other complications and limited harvest amount from intraoral autogenous bone acts as a limitation ${ }^{5-7)}$. Many graft materials have been developed to substitute autogenous bone. Allogenic bone shows active osteoconductive ability; and depending on the treatment method, osteoinductivity can be shown but it is hard to expect new bone formation effects. Also one cannot completely exclude the possibility of disease spreading and the low bone formation effect makes it difficult to equal the safety and validity of autogenous bone ${ }^{8,9}$. Commercial bone graft material based on allogenic bone that is produced through certain treatment processes is widely used clinically, but only osteoconduction without new bone formation and osteoinduction can be expected and it is not absorbed into tissue and remains for a long time. This implies that it is unclear whether the grafted material participated in bone remodeling through new bone formation and also the fact that it is hard to completely exclude the possibility of disease spreading with this material is a disadvantage ${ }^{10)}$. The recent worries concerning bovine spongiform encephalopathy has increased the repulsion in using allergenic bone. Synthetic bone graft material of calcium phosphate type including hydroxyapatite (HA) and tricalcium phosphate are commonly used. Synthetic bone is cheap since mass production is possible without limits in harvesting and there is no danger of disease spreading but it only acts as a scaffold so only osteoconductivity can be expected ${ }^{11)}$. For such reasons, many studies concerning the development of a bone graft material that can substitute for autogenous bone have been conducted but the impact was not significant previously. However the recent development of autogenous tooth bone graft material in Korea is raising expectations as a substitute for autogenous bone graft material ${ }^{3)}$

Autogenous tooth bone graft material is produced by extracting bone components from autogenous teeth and shows almost identical constituents as bone. The material is autogenous tissue, which eliminates immune and foreign body reaction so it shows excellent biocompatibility without the possibility of disease spreading which acts as an advantage for the patients to accept without psychological rejection reactions. In this study we used mini-pigs to evaluate the bone healing ability of autogenous tooth bone graft material.

Pigs are difficult to handle, and they grow rapidly and weigh too much when grown up, so it is considered inappropriate as an experimental animal in the field of dentistry. But the development of mini-pigs solved such problems at once and the application of mini-pigs to dental studies has opened a new era for studies using pigs. The constituents of porcine bone are highly similar to human bone making it very useful as an experimental animal for preclinical studies $^{12)}$. The intensity of bony trabecule is denser compared to human but the fact that the bone regeneration rate is closer to human compared to canine works as an advantage in establishing conditions such as in humans. This study evaluated the bone healing ability of autogenous tooth bone graft material based on extracted autogenous teeth in a mini-pig cranial defect model through histologic examinations and osteonectin reverse transcription polymerase chain reaction (RT-PCR) quantitative analysis.

\section{Materials and Methods}

\section{Study Materials}

\section{1) Experimental Animal}

Six healthy male mini-pigs (mean weight $39 \mathrm{~kg}, 24$ months after birth, Prestige World Genetics, Pyeongtaek, Korea) that had been raised under identical conditions for a certain period were used as experimental animals in this study.

\section{2) Graft Material}

Autogenous tooth bone graft material produced from teeth extracted from the experimental animals were used as graft material with each mini-pig receiving autogenous tooth bone graft material originating from itself. 


\section{Study Method}

1) Animal Experiment

(1) Extraction

Atropine (Kwangmyung Pharmaceutical Ind. Co., Ltd., Seoul, Korea), xylazine (Rompun, Bayer Korea Co., Seoul, Korea) and tiletamine-zolazepam (Zoletil, Virbac Animal Health, Carros, France) were intramuscularly injected into selected mini-pigs as pre-anesthesia treatment, then Enflurane (Gerolan, ChoongWae Pharmaceutical Co., Seoul, Korea) was administered for inhalation anesthesia. Infiltration anesthesia was done in the site of surgery for anesthesia and hemostasis with lidocaine (2\% lidocaine $\mathrm{HCl}$-epinephrine, $1.8 \mathrm{ml}$, Yuhan Co., Seoul, Korea). The first, second, and third mandibular premolar were extracted and treated for autogenous tooth bone graft material. The flap was pulled to position and sutured with absorbable silk suture after extraction and the operation was completed.

Antibiotics were injected intramuscularly right after the operation and a fluid diet was provided to protect the surgical site until wound healing was considered visibly complete.

(2) Autogenous tooth material graft to cranial defect Under the previously described anesthesia, the cranium of the mini-pig was incised and dissected to expose the cranium. Three bony defects with a diameter and depth of 10 $\mathrm{mm}$ and $4 \mathrm{~mm}$ each were formed on both the left and right for a total of 6 defective areas per pig. The left side was the experimental group with autogenous tooth material grafted and the right side was used as control without graft material. A flap was pulled to position and sutured with absorbable silk suture and the operation was completed. Antibiotics were injected intramuscularly right after the operation as after tooth extraction.

\section{2) Production of Autogenous Tooth Bone Graft Material}

The extracted tooth was put in $70 \%$ ethyl alcohol and sent to a professional treatment facility (Korea Tooth Bank Co., Seoul, Korea). The tooth was separated into crown and root portion after foreign substances such as attached soft tissue and calculus was removed before pulverization. The particles were ground to a size of $1 \sim 2 \mathrm{~mm}$ and then cleansed in an ultrasonic cleanser with distilled water and hydrogen peroxide solution to remove remaining contaminants. The cleansed particles were dehydrated with ethyl-alcohol and then defatted with ethyl ether solution. The particles that completed the above procedure underwent a lyophilization process and were gas sterilized with ethylene oxide. The particles were then packaged and sent to the laboratory in this state for grafting.

\section{3) Tissue Specimen Production and Analysis}

The experimental animals were sacrificed at 4, 8, and 12 weeks after grafting and the defect area was removed as a block including the surrounding tissue to produce tissue specimens following the conventional method. Hematoxylin-eosin and Masson's trichrome were used for staining. The specimen was observed histopathologically using a light microscope (Olympus BX-51, Olympus Co., Tokyo, Japan).

\section{4) Osteonectin Real-time RT-PCR}

A 7- $\mu \mathrm{m}$ thick deparaffinized tissue section was harvested from each tissue and total RNA was extracted using FFPE RNA extraction kit (QIAGEN Korea, Seoul, Korea), then cDNA was also synthesized using a RT-PCR kit (iNtRON Biotechnology, Seongnam, Korea) and PCR was done under the following conditions. Denaturalization for 10 minutes at $95^{\circ} \mathrm{C}, 30$ seconds at $95^{\circ} \mathrm{C}, 30$ seconds at $60^{\circ} \mathrm{C}$, and 30 seconds at $72^{\circ} \mathrm{C}$, then this cycle was repeated 40 times. The expression of the housekeeping gene glyceraldehyde 3- phosphate dehydrogenase (GAPDH) was used as a control. The relative quantitative analysis of osteonectin gene expression was calculated as $2^{-\Delta \Delta C \mathrm{~T}}$. The $\Delta \Delta C_{\mathrm{T}}$ is calculated by subtracting the housekeeping gene GAPDH $C_{\mathrm{T}}$ value from the osteonectin $C_{\mathrm{T}}$ value of tumor tissue then subtracting the value calculated by subtracting the housekeeping gene GAPDH $C_{\mathrm{T}}$ value from the osteonectin $C_{\mathrm{T}}$ value of normal tissue from the initial value. The equation is as below.

$\Delta \Delta C_{\mathrm{T}}=\left(C_{\mathrm{T}, \text { osteonectin }}-C_{\mathrm{T}, \mathrm{GAPDH}}\right)$ tumor tissue- $\left(C_{\mathrm{T}, \text { osteonectin }}-C_{\mathrm{T}, \mathrm{GAPDH}}\right)$ normal tissue

The primer sequence of osteonectin and GAPDH is presented in Table 1 .

Table 1. Primer sequence for the osteonectin and GAPDH

\begin{tabular}{ccc}
\hline Primer & Primer sequence $\left(5^{\prime}-3^{\prime}\right)$ & $\begin{array}{c}\text { Amplicon } \\
\text { size }\end{array}$ \\
\hline Osteonectin & CAAGAACGTCCTGGTCACCTTCGCTTCTCATTCTCGT- & $102 \mathrm{bp}$ \\
GGATC & \\
GAPDH & GCTTTGCCCCGCGATCTAATGTTCGCCAAATCCGTTC- & $90 \mathrm{bp}$ \\
& ACTCCGACCTT & \\
\hline
\end{tabular}

GAPDH: glyceraldehyde 3-phosphate dehydrogenase. 


\section{Statistical Analysis}

Wilcoxon's rank sum test was conducted to statistically compare the significance of RT-PCR values of the two groups. The results were considered significant with $\mathrm{P}<$ 0.001 .

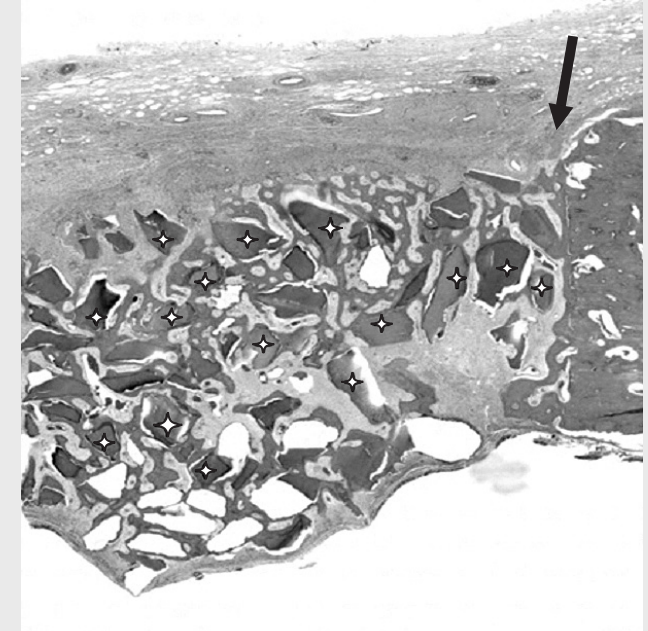

Figure 1. Experimental group of 4 weeks. Extensive bone formation around the tooth powder granules. Arrow indicates the boundary between calvaria bone and bur hole. Hematoloxylin and eosin staining ( $\times 100)$.

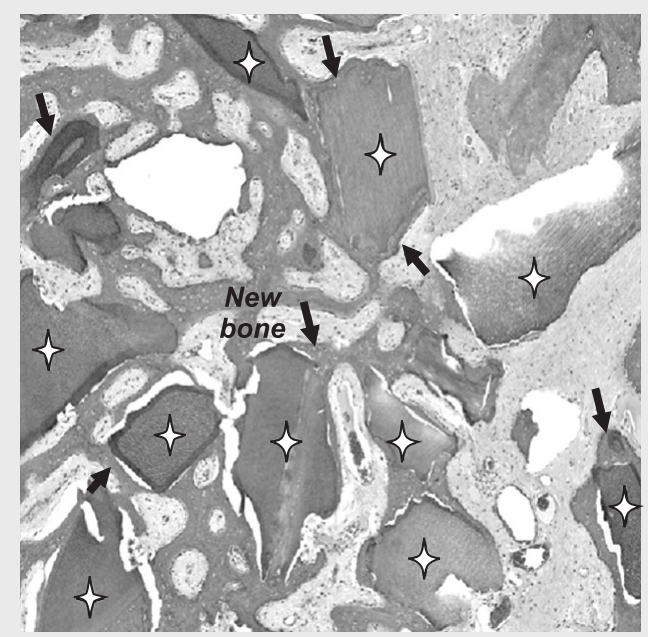

Figure 2. Experimental group of 4 weeks. Bone formation of the bur hole. Asterisks and arrows indicate graft tooth granule materials and new bone formation around the tooth granules, respectively. Hematoloxylin and eosin staining $(\times 100)$.

\section{Result}

\section{Histologic Findings}

Bone formation surrounding the tooth powder granule was observed at 4 weeks in the experimental group where the bur hole was filled with autogenous tooth bone graft material (Figs. 1 and 2). However, the inside of the bur hole

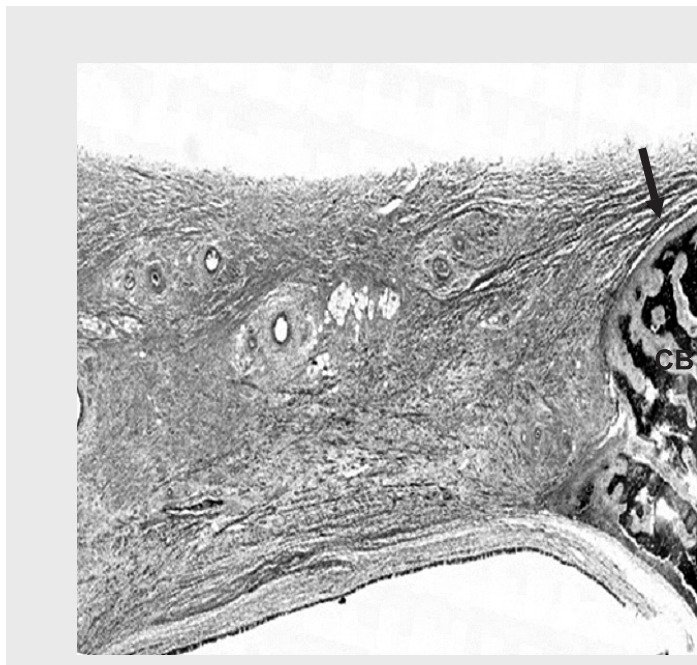

Figure 3. Control group of 4 weeks. Extensive fibrosis of the bur hole. The arrows indicate boundary between calvaria bone and bur hole. Masson's trichrome staining (x100). CB: calvaria bone.

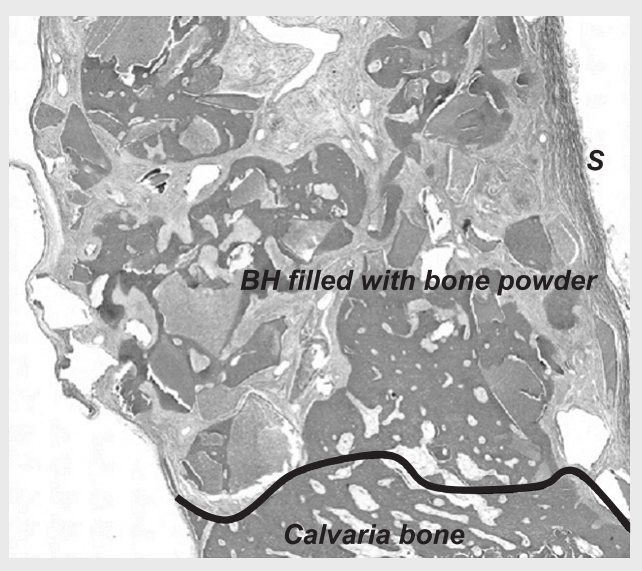

Figure 4. Experimental group of 8 weeks. Extensive new bone formation around the tooth powder granules. Line indicates between bur hole and calvaria bone. Hematoloxylin and eosin staining $(\times 100)$. BH: bur hole. 
showed fibrosis and there was no sign of bone formation in the control group (Fig. 3). Such bone formation was different according to the amount of graft material, with more graft material showing more bone formation compared to less graft material. Bone formation was increased at 8 weeks compared to 4 weeks in the experimental group (Figs. 4 and 5). Only fibrosis could be observed in the control group until then (Fig. 6). At 12 weeks, active bone formation was found around the graft material in the experimental group (Figs. 7 and 8) while only fibrosis could be observed in the control group (Fig. 9).

\section{RT-PCR Results}

The RT-PCR results of osteonectin for tissue specimens at 4,8 , and 12 weeks showed a higher value in the experimental group compared to the control group regardless of time (Table 2, Fig. 10).

\section{Discussion}

After the first study by Yeomans and Urist ${ }^{2}$ that reported bone formation after applying decalcified teeth to areas that are not bone ${ }^{2)}$ it is true that interest in studies concerning the bone forming ability of teeth have been scant until now. But the recent reports of Kim et al. ${ }^{3}$ that teeth can be successfully applied as graft material after appropriate treatment and the report from Murata et al. ${ }^{4)}$ of Japan were all results of ideas based on previous studies that show the constituents of tooth and bone are similar.

Autogenous bone grafting is known as the gold standard for graft procedures and is considered the most ideal. As already known, autogenous bone shows osteoconductivity and also osteoinduction can be expected from the rich bone

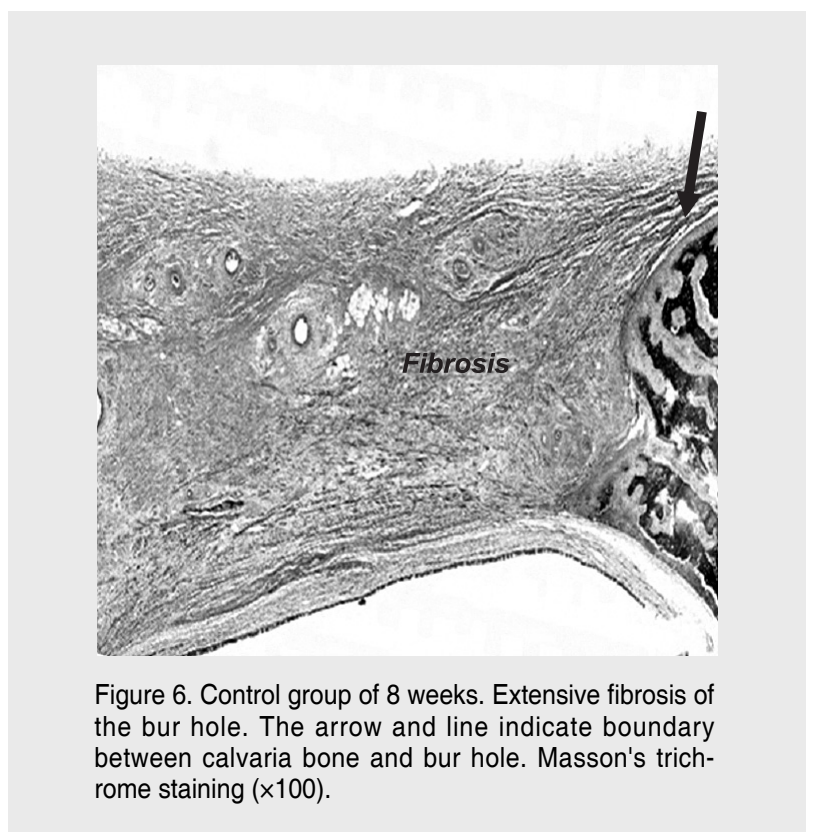

Figure 6. Control group of 8 weeks. Extensive fibrosis of the bur hole. The arrow and line indicate boundary calvaria bone and bur hole. Masson's trich-
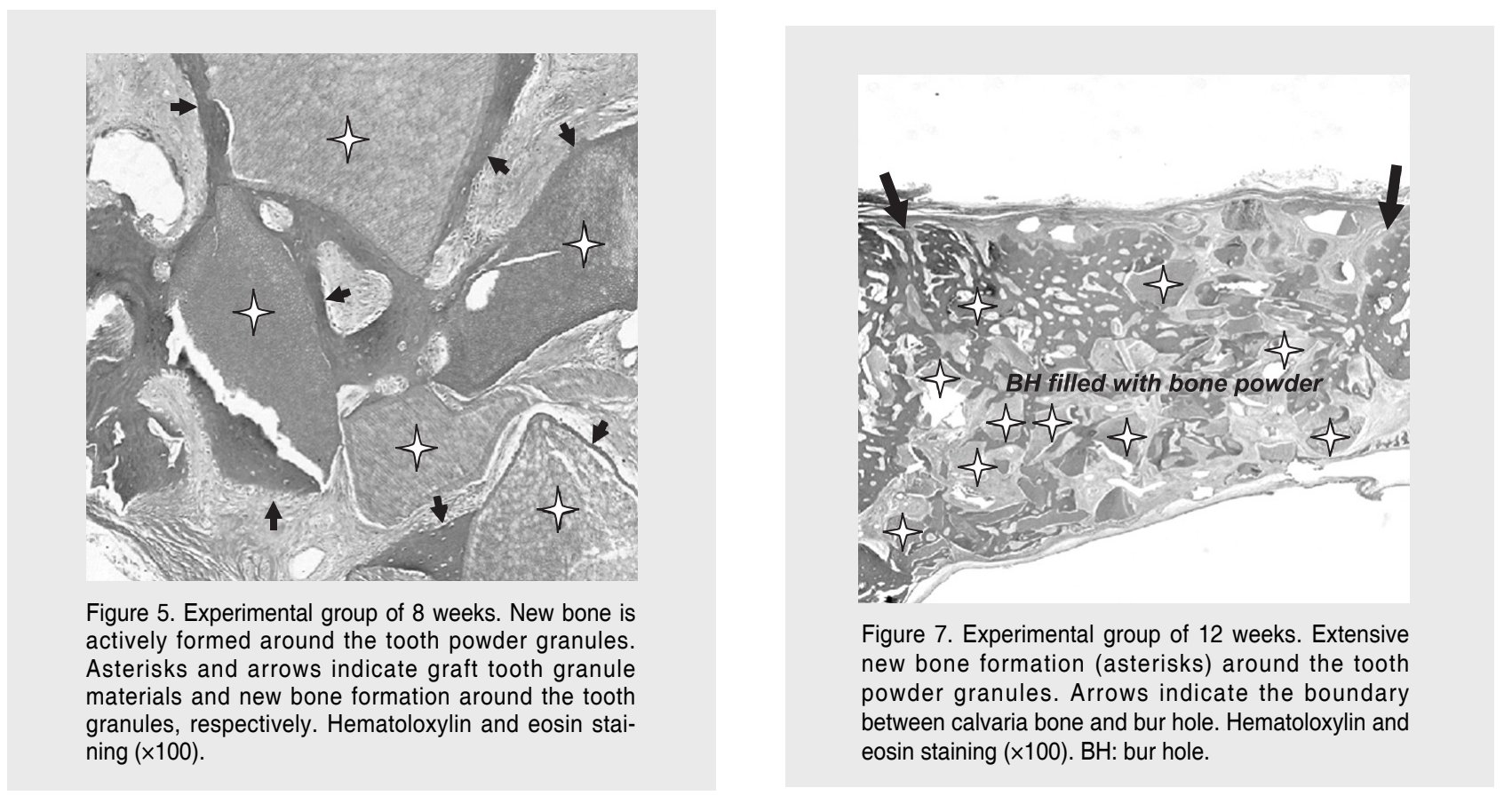
morphogenic protein included in the cortical bone. Furthermore new bone formation effects can be anticipated from the stem cells that are known to exist in bone marrow when this part is included in the graft material. Autogenous tooth bone graft material shows highly similar constituents as bone. This allows it to have the advantages of bone, making it a new concept for graft material with excellent

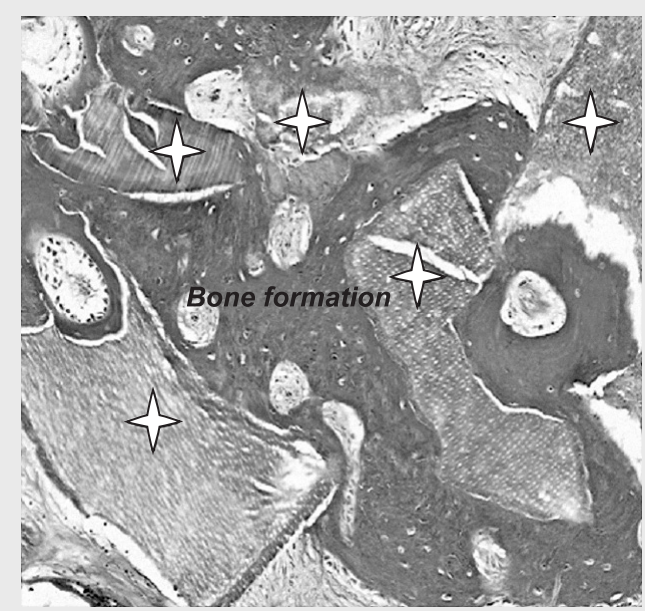

Figure 8. Experimental group of 12 weeks. Extensive new bone formation is noted around the bone powder granules in the bur hole. Asterisks indicates tooth powder materials. Hematoloxylin and eosin staining $(\times 100)$. clinical applications. Since it is autogenous, it has excellent biocompatibility with no immune or foreign body reaction and the psychologic rejection reaction of patients can be eliminated since there is no possibility of disease spreading, unlike allogenic and heterogenous bone grafting.

Numerous previous studies showed that the dentin, cementum, and periodontal ligament of the tooth contain many growth factors that are effective in bone formation. Ike and Urist $^{13)}$ showed that BMP-2 has important osteoinductive effects for bone formation and dentin contains growth factors such as insulin-like growth factor-II (IGF-II), BMP-2, and transforming growth factor (TGF)-beta that are similar to bone ${ }^{14)}$. Cementum and cementoblasts are known to contain TGF-beta, IGF-I, and platelet-derived growth factor $(\mathrm{PDGF})^{15)}$. Emecen et al. ${ }^{16)}$ reported that periodontal ligament also contains substances such as TGF-beta, IGF-I, basic fibroblast growth factor, vascular endothelial growth factor, BMP-2, PDGF, and type I collagen. Previous studies based on tooth ash powder that had every organic com-

\begin{tabular}{llll}
\multicolumn{4}{l}{ Table 2. Osteonectin RT-PCR analysis } \\
\hline & 4 week & 8 week & 12 week \\
\hline $2^{-\triangle C T}$ (experimantal) & 0.0842 & 0.067 & 0.0748 \\
$2^{-\triangle M C T}$ (control) & 0.0018 & 0.0015 & 0.0017 \\
\hline
\end{tabular}

RT-PCR: reverse transcription polymerase chain reaction.

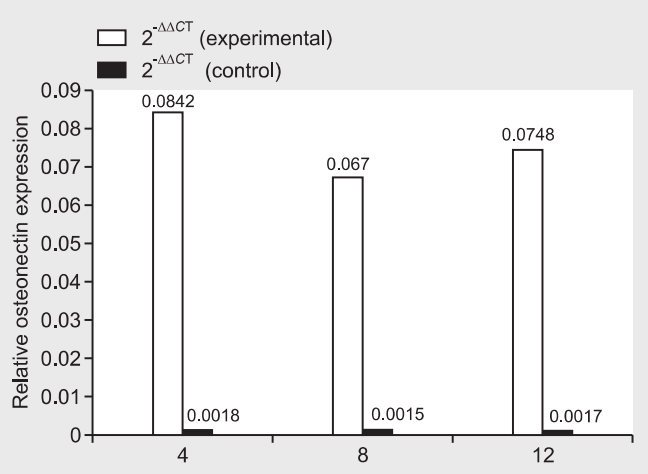

Figure 10. Osteonectin RT-PCR analysis shows increased osteonectin expression in the experimental group all stages of the osteoblastic development contrary to the control group $(\mathrm{P}<0.001)$. RT-PCR: reverse transcription polymerase chain reaction. 
ponent eliminated due to the possibility of infection and disease spreading showed that the loss of organic substances that have osteoinductivity resulted in limited regeneration of alveolar bone ${ }^{17-22)}$ but the current autogenous tooth bone graft material that has both organic and inorganic substances reserved allows us to expect not only osteoconductivity but also osteoinductivity ${ }^{3)}$.

As the first bone substrate protein to be extracted without using a resolvent catalyst, osteonectin can be found in various cells; but in the case of adult cells, it can typically be found in calcified tissue ${ }^{23)}$.

Osteonectin was also used as an index for calcification tendency in this study and the experimental group with autogenous tooth bone graft material showed a higher calcification tendency that was statistically significant compared to the control group. When quantitatively evaluating the expression of osteonectin, the $2^{-\triangle \Delta C T}$ method is generally used due to its convenience because the measured data is normalized as a relative value of the endogenous reference ${ }^{24)}$. Further studies of various calcified tissue indices including osteonectin will enable more valuable studies concerning the bone healing ability of autogenous tooth bone graft material.

Nampo et al. ${ }^{25)}$ compared the bone formation ability of autogenous tooth bone graft material and autogenous bone harvested from the long bone of rats in a mandibular defect area and reported that autogenous tooth bone graft material showed better bone formation compared to long bone. Studies based on autogenous tooth bone graft material as experimental and HA as control material grafted to a mandibular bony defect of mini-pigs reported that the experimental group showed better initial bone formation and histometric evaluations showed that the experimental group had better quantitative results ${ }^{26}$. Mini-pigs were also used in this study because the basic alveolar bone components of mini-pigs are similar to human so it is widely used as an experimental animal in bone grafting studies in dentistry ${ }^{27}$.

The results of this study show that the control group without any bone graft material showed a tendency of fibrosis without any new bone formation while active bone formation was observed in the experimental group with autogenous tooth bone graft material. We report the results as experimental data that supports the bone healing ability of autogenous tooth bone graft material along with the osteonectin bone formation index study results that show statistically significant calcification tendency of the experimental group with autogenous tooth bone graft material compared to the control.

The small study size and short follow-up period may be considered as a limitation of this study and additional studies are necessary. But considering that extracted teeth that have been discarded until now can be recycled along with the additional advantage of decrease in donor site morbidity, autogenous tooth bone grafting seems to be of value as a new bone grafting method that may equal conventional bone grafting.

\section{Conclusion}

In conclusion, active bone regeneration ability was observed in the case of autogenous tooth bone graft material grafted to a bony defect. We believe this material will show better results when applied in a clinical setting.

434-7.

5. Hiatt WH, Schallhorn RG. Intraoral transplants of cancellous bone and marrow in periodontal lesions. J Periodontol. 1973; 44: 194-208.

6. Ellegaard B, Karring T, Davies R, Löe H. New attachment after treatment of intrabony defects in monkeys. J Periodontol. 1974; 45: 368-77.

7. Schallhorn RG. Present status of osseous grafting procedures. J Periodontol. 1977; 48: 570-6.

8. Quattlebaum JB, Mellonig JT, Hensel NF. Antigenicity of freeze-dried cortical bone allograft in human periodontal osseous defects. J Periodontol. 1988; 59: 394-7.

9. Schwartz Z, Mellonig JT, Carnes DL Jr, de la Fontaine J, Cochran DL,
3. Kim YK, Kim SG, Byeon JH, Lee HJ, Um IU, Lim SC, Kim SY. Development of a novel bone grafting material using autogenous teeth. Oral Surg Oral Med Oral Pathol Oral Radiol Endod. 2010; 109: 496503.

4. Murata M, Akazawa T, Takahata M, Ito M, Tazaki J, Hino J, Nakamura K, Iwasaki N, Shibata T, Arisue M. Bone induction of human tooth and bone crashed by newly automatic mill. J Ceram Soc Jpn. 2010; 118: 


\section{References}

Dean DD, Boyan BD. Ability of commercial demineralized freeze-dried bone allograft to induce new bone formation. J Periodontol. 1996; 67: 918-26.

10. Sogal A, Tofe AJ. Risk assessment of bovine spongiform encephalopathy transmission through bone graft material derived from bovine bone used for dental applications. J Periodontol. 1999; 70: 1053-63.

11. Han T, Carranza FA Jr, Kenney EB. Calcium phosphate ceramics in dentistry: a review of the literature. J West Soc Periodontol Periodontal Abstr. 1984; 32: 88-108.

12. Thorwarth M, Schultze-Mosgau S, Kessler P, Wiltfang J, Schlegel KA. Bone regeneration in osseous defects using a resorbable nanoparticular hydroxyapatite. J Oral Maxillofac Surg. 2005; 63: 1626-33.

13. Ike M, Urist MR. Recycled dentin root matrix for a carrier of recombinant human bone morphogenetic protein. J Oral Implantol. 1998; 24: $124-32$

14. Gao J, Symons AL, Bartold PM. Expression of transforming growth factor-beta 1 (TGF-beta1) in the developing periodontium of rats. J Dent Res. 1998; 77: 1708-16.

15. Saygin NE, Tokiyasu Y, Giannobile WV, Somerman MJ. Growth factors regulate expression of mineral associated genes in cementoblasts. J Periodontol. 2000; 71: 1591-600.

16. Emecen P, Akman AC, Hakki SS, Hakki EE, Demiralp B, Tözüm TF, Nohutcu RM. ABM/P-15 modulates proliferation and mRNA synthesis of growth factors of periodontal ligament cells. Acta Odontol Scand. 2009; 67: 65-73.

17. Kim SG, Yeo HH, Kim YK. Grafting of large defects of the jaws with a particulate dentin-plaster of paris combination. Oral Surg Oral Med Oral Pathol Oral Radiol Endod. 1999; 88: 22-5.

18. Kim SG, Chung CH, Kim YK. Grafting defects using a particulate dentin-laster of Paris combination for implant placement: a case report.
Hosp Dent (Tokyo). 2001; 13: 127-30.

19. Kim SG, Chung CH, Kim YK, Park JC, Lim SC. Use of particulate dentin-plaster of Paris combination with/without platelet-rich plasma in the treatment of bone defects around implants. Int J Oral Maxillofac Implants. 2002; 17: 86-94.

20. Hwang YJ, Kim SG, Yoon JH, Lim SC. Effect of the bone regeneration of the mixture of human, bovine, pig, rabbit, or dog toosh-ash and the plaster of Paris in rats. J Korean Assoc Maxillofac Plast Reconstr Surg. 2004; 26: 155-61

21. Park SS, Kim SG, Lim SC, Ong JL. Osteogenic activity of the mixture of chitosan and particulate dentin. J Biomed Mater Res A. 2008; 87: $618-23$.

22. Su-Gwan K, Hak-Kyun K, Sung-Chul L. Combined implantation of particulate dentine, plaster of Paris, and a bone xenograft (Bio-Oss) for bone regeneration in rats. J Craniomaxillofac Surg. 2001; 29: 282-8.

23. Robey PG: Bone matrix proteoglycans and glycoproteins. In: Bilezikian JP, Raisz LG, Rodan GA. Principles of bone biology. vol.1. London: Academic Press; 2002. p. 225-37.

24. Livak KJ, Schmittgen TD. Analysis of relative gene expression data using real-time quantitative PCR and the 2(-Delta Delta C(T)) Method. Methods. 2001; 25: 402-8

25. Nampo T, Watahiki J, Enomoto A, Taguchi T, Ono M, Nakano H, Yamamoto G, Irie T, Tachikawa T, Maki K. A new method for alveolar bone repair using extracted teeth for the graft material. J Periodontol. 2010; 81: 1264-72.

26. Jeong HR, Hwang JH, Lee JK. Effectiveness of autogenous tooth bone used as a graft material for regeneration of bone in miniature pig. J Korean Assoc Oral Maxillofac Surg. 2011; 37: 375-9.

27. McKee MD, Aoba T, Moreno EC. Morphology of the enamel organ in the miniature swine. Anat Rec. 1991; 230: 97-113. 